\title{
CARCINOGENIC OESTROGENS INDUCE RESPIRATION DEFICIENCY MUTATION IN YEAST
}

\author{
H. STOPPER* and M. METZLER $†$ \\ *Institute of Pharmacology and Toxicology, University of Würzburg, Versbacher Strasse 9, \\ D-8700 Würzburg and †Department of Food Chemistry and Environmental Toxicology, University of \\ Kaiserslautern, Erwin-Schrödinger-Strasse, D-6750 Kaiserslautern, Germany
}

\begin{abstract}
In addition to hormonal activity, genetic damage has been proposed as an important factor in oestrogen-mediated carcinogenesis. However, as short-term tests for oestrogens usually fail to show DNA mutations, lesions other than classic nuclear DNA mutation have to be considered. Oestrogeninduced mitochondrial damage was studied in the yeast Saccharomyces cerevisiae. Stilbene-type, but not steroidal, oestrogens were found to induce respiration-deficient petite mutation. The effect was inversely correlated with cytotoxicity and required aromatic hydroxyl groups at the stilbene molecule. It only occurred under growth conditions and apparently was not due to the ATPase inhibitory qualities of stilbene oestrogens. Other studies have shown that petite mutation clones, which can be induced by a variety of substances, contain altered mitochondrial DNA. The mechanism of petite mutation induction might be important in tumorigenesis by also acting on nuclear DNA or facilitating carcinogenesis by disturbance of mitochondrial function.
\end{abstract}

\section{Introduction}

Certain oestrogens induce cancer in laboratory animals and are associated with tumour formation in man, but the mechanism of oestrogen-mediated carcinogenesis is still unclear (IARC, 1979). Syrian hamster embryo fibroblasts, which do not have measurable amounts of oestrogen receptors (McLachlan et al., 1982) may be neoplastically transformed by a variety of synthetic and natural oestrogens (McLachlan et al., 1982; Tsutsui et al., 1987). The transformation occurs in the absence of detectable point mutations (Tsutsui et al., 1987). In addition to their tumour-promoting abilities in hormone target tissues, oestrogens appear to induce genetic damage that is different from classic gene mutations, but that also gives rise to neoplastic transformation and possibly cancer.

To investigate oestrogen-mediated damage, mechanisms that do not involve direct interaction with nuclear DNA have to be considered. Mitochondria, as possible targets, can easily be studied in the yeast Saccharomyces cerevisiae. When grown in the presence of a variety of chemicals, $S$. cerevisiae becomes deficient in respiration, while still retaining the ability to produce energy by fermentation. These so-called petite mutants (Mahler and Perlman, 1973) have been found to contain altered mitochondrial DNA. When we investigated oestrogens several, but not all of them, induced high frequencies of petite mutants in the diploid yeast strain D61.M. Many non-hormonal petite mutation inducers are generally known to interact with DNA and therefore may interfere with mitochondrial DNA-replication/transcription or may

Abbreviations: $\mathrm{DES}=$ diethylstilboestrol; $3,3^{\prime}$-DES $=3,3^{\prime}$ diethylstilboestrol; DMSO = dimethylsulphoxide; $\mathrm{PBS}=$ phosphate buffered saline; SDS = sodium dodecyl sulphate; tetrafluoro-DES = tetrafluoro-diethylstilboestrol; Z,Z,-DIES = Z,Z,-dienoestrol. cause direct mitochondrial DNA damage. As oestrogens have not been shown conclusively to interact with DNA, and as oestrogen-mediated tumorigenesis also seems to involve targets other than DNA, elucidation of the mechanism by which oestrogens induce petite mutation might also help to clarify the processes involved in cell transformation.

The data provided in this report show that petite mutation induction by oestrogens is highly dependent on the stilbene structure as well as the presence of hydroxyl groups located on the benzene rings.

\section{Materials and Methods}

Yeast strain and growth medium. The diploid yeast strain D61.M (Zimmermann and Scheel, 1984) was kindly provided by Professor Zimmermann (Darmstadt, Germany). The standard $1 \%$ yeast extract, $2 \%$ peptone and $2 \%$ glucose medium was used for routine maintenance and experiments. Respiration-deficient clones were confirmed by their inability to grow on medium containing glycerol instead of glucose as the carbon source. Media were solidified with $2 \%$ Difco agar when necessary.

Induction of petite mutants. $1 \times 10^{6}$ exponentially growing cells were inoculated per $\mathrm{ml}$ of complete growth medium in test-tubes. Test compounds (dissolved in DMSO to give a final DMSO concentration of $2 \%$ or less) or solvent controls were added. After overnight incubation at $28^{\circ} \mathrm{C}$ in a shaking waterbath, 1000 cells were plated on complete growth medium (two plates per experiment). D61.M carries the ade 2 mutation. This mutation leads to the accumulation of a red pigment if the cells are grown aerobically and are mitochondrially proficient. Petite mutants do not produce the red pigment and are a creamy-white colour. After scoring the plates for the total number of colonies (to estimate viability) and the number of white colonies, up to 50 white colonies were tested on glycerol-containing medium 


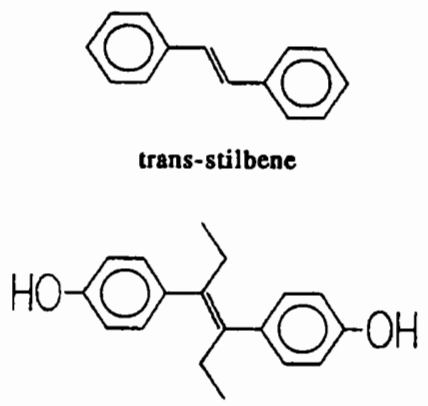

diethylstilboestrol (DES)

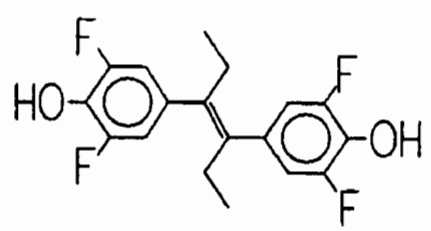

tetrafluoro-DES

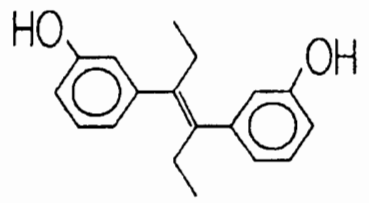

3,3'-diethylstilboestrol

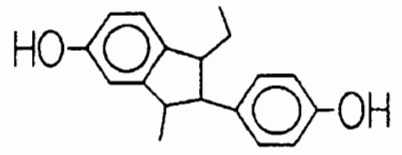

indanoestrol

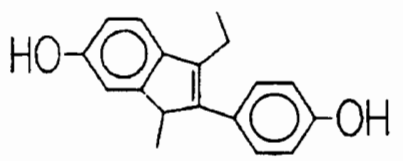

indenoestrol $\mathbf{A}$

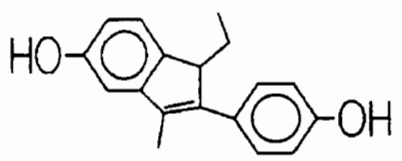

indenoestrol $\mathbf{B}$

Fig. 1. Chemical structures of the stilbene-type oestrogens used in this study.

to confirm their petite mutation status. A few colonies turned out to be white for reasons other than petite mutation, for example mutation or recombination in the ade 2 locus. If not more than three such colonies were found, no correction was made in the evaluation of white colonies as petite mutants. The benzamidine derivative berenil (Mahler and Perlman, 1973) was used as a positive control for petite mutation induction.

Oestrogen concentrations. The solubility of oestrogens in buffer is about $10 \mu \mathrm{g} / \mathrm{ml}$. Due to unspecific binding in culture medium, microscopically or macroscopically visible crystals appear only at much higher concentrations (about $100 \mu \mathrm{g} / \mathrm{ml}$ ). Using 'non-soluble' concentrations seems justified as long as an increase in concentration also produces an increase in biological effect. We employed concentrations up to $100 \mu \mathrm{g} / \mathrm{ml}$ and considered any higher substance concentration as a 'saturated solution'.

\section{Results and Discussion}

The ability of various stilbene-type (Fig, 1) and steroidal oestrogens to induce respiration deficiency petite mutation under conditions permitting growth was investigated. Figure 2 shows the data obtained with $3,3^{\prime}$-diethylstilboestrol $\left(3,3^{\prime}\right.$-DES) as a typical representative for stilbene oestrogens. The percentage of petite mutation colonies was inversely correlated with viability. This was not the case for the nonhormonal petite mutation inducer berenil, which did not affect viability. The maximal percentage of petite mutation colonies induced by $3,3^{\prime}$-DES was $63 \%$ at $80 \mu \mathrm{g} / \mathrm{ml}$, a percentage that could be induced with $6 \mu \mathrm{g} / \mathrm{ml}$ berenil.

A diagram equivalent to the one shown in Fig. 2 for 3,3'-DES was established for each substance tested. From these diagrams were calculated the substance concentrations at which the relative viabilities were 20 and 50 compared with the control value. Percent petite mutation colonies for these substance concentrations were then also calculated from the diagrams. These values are listed in Table 1: the synthetic stilbene oestrogens 3,3'-DES, tetrafluorodiethylstilboestrol (tetrafluoro-DES), indenoestrol $A$, indenoestrol $B$ and indanoestrol were potent inducers of petite mutation (in combination with a pronounced cytotoxic effect). Their dose-response curves for petite mutation induction and viability (data not shown) were similar to that of $3,3^{\prime}$-DES. Z,Z,-dienoestrol (Z,Z-DIES), a metabolite of the synthetic stilbene oestrogen diethylstilboestrol (DES) was a much weaker inducer of petite mutants

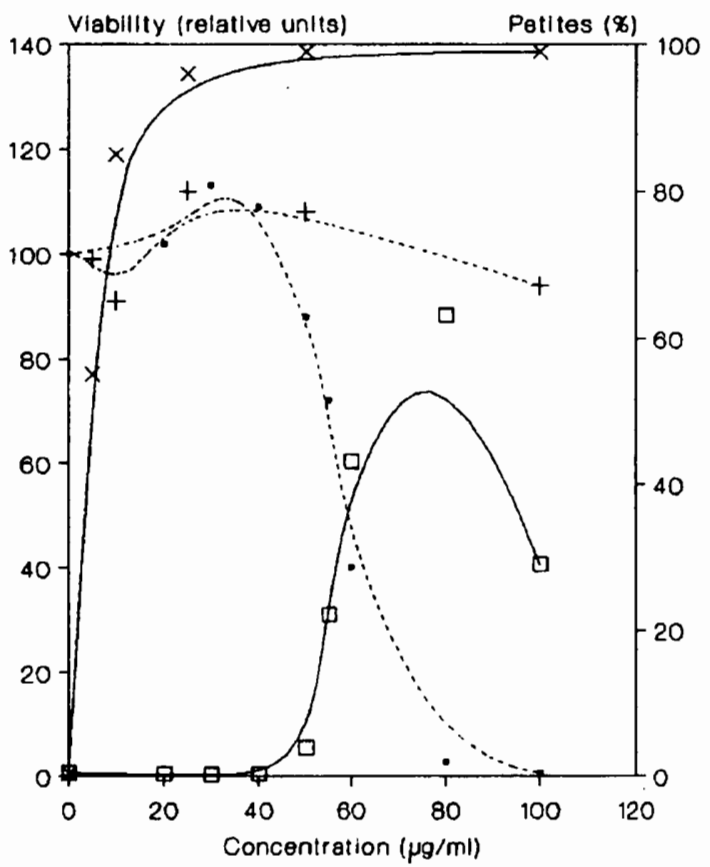

Fig. 2. The effect of 3,3'-DES on the induction of petite mutation colonies (solid line - $\square-$ ) and relative viability (dotted line, - - - . Berenil was used as a positive control ( $\%$ petites $-x-$, viability -+- ). The percentage of colonies expressing the petite mutation is shown as 'petites'. The number of surviving colonies compared with the control is shown as 'viability'. 
Table 1. Induction of petite mutation colonies and roduction of relative viability by oestrogens under growth conditions. Substances were incubated overnight. Controls (solvent only) gave $0.26 \%$ petite mutation colonies as an average of 11 experiments. Relative viability was set to 100 for the control carried out with cach substance. Values in this table were calculated from diagrams for cach substance equivalent to the one shown in Fig. 2 for 3,3'-DES. Where viability was not reduced, petite mutation values at a concentration of $100 \mu$ g/ml and at saturated solution are given for comparison

\begin{tabular}{|c|c|c|c|c|c|c|c|c|}
\hline \multirow{4}{*}{$\frac{\text { Substance }}{3,3^{\prime} \text {-DES }}$} & \multicolumn{2}{|c|}{$\begin{array}{l}\text { Conch }(\mu \mathrm{g} / \mathrm{ml}) \text { at } \\
\text { relative viability }\end{array}$} & \multirow{2}{*}{\multicolumn{2}{|c|}{$\begin{array}{c}\text { Relative viability } \\
\text { at concn }(\mu \mathrm{g} / \mathrm{ml}) \\
100 \text { saturated solution }\end{array}$}} & \multicolumn{2}{|c|}{$\begin{array}{l}\text { Petites }(\%) \text { at } \\
\text { relative viability }\end{array}$} & \multirow{2}{*}{\multicolumn{2}{|c|}{$\begin{array}{c}\text { Petites }(\%) \text { at } \\
\text { concn }(\mu \mathrm{g} / \mathrm{m}) \\
100 \text { saturated solution }\end{array}$}} \\
\hline & \multirow{3}{*}{$\begin{array}{l}20 \\
73 \\
22\end{array}$} & \multirow{2}{*}{$\frac{50}{59}$} & & & 20 & 50 & & \\
\hline & & & 0.6 & 0 & 52 & 35 & 29 & - \\
\hline & & 17 & 0 & 0 & 77 & 71 & - & - \\
\hline Z,Z-DIES & - & 175 & 89 & 30 & - & 30 & 2 & 30 \\
\hline DES & - & - & 90 & 108 & - & - & 0.2 & 0.2 \\
\hline Indenoestrol A & 34 & 29 & 0 & 0 & 30 & 10 & - & - \\
\hline Indenoestrol B & 65 & 55 & 0 & 0 & 58 & 25 & - & - \\
\hline Indanoestrol & 66 & 54 & 0 & 0 & 64 & 28 & - & - \\
\hline Stilbene & - & - & 94 & 102 & - & - & 0.4 & 0.5 \\
\hline $17 \beta$-Oestradiol & - & - & 108 & 110 & - & - & 0.8 & 0.9 \\
\hline Oestrone & - & - & 94 & 107 & - & - & 0.2 & 0.2 \\
\hline Berenil & - & - & 94 & nd & - & - & 99 & nd \\
\hline
\end{tabular}

nd $=$ not determined

and relative viability did not decrease below 30 , even in saturated solution. DES, trans-stilbene (which does not possess phenolic hydroxyl groups) and the natural steroids oestrone and $17 \beta$-oestradiol did not show any effect. Berenil (positive control) induced $99 \%$ petite mutation colonies without loss of viability.

In another set of experiments (Table 2) $40 \mu \mathrm{g} / \mathrm{ml}$ of the membrane active agent sodium dodecyl sulphate (SDS) was added before overnight incubation for those substances that induced petite mutation at concentrations higher than $100 \mu \mathrm{g} / \mathrm{ml}$ or not at all. Under these conditions Z,Z,-DIES and DES showed cytotoxicity similar to the active substances in Table 1 and percentages of petite mutants also increased. In contrast, trans-stilbene and the steroids oestrone and $17 \beta$-oestradiol still did not show any effect.

Oestrogenic activity alone did not appear to be sufficient for the induction of petite mutation. The effect was limited to stilbene oestrogens and required the hydroxyl groups on the benzene rings. There was no correlation with the ability to form quinoid substances. These have been shown to bind to proteins (Epe et al., 1990) and to occur in oestrogen metabolism (Metzler, 1984). As indenoestrol B, indanoestrol, Z,Z-DIES and 3,3'-DES are not able to form quinoid structures, binding of metabolically activated quinoid structures to proteins (e.g. enzymes of the mitochondrial DNA replication/transcription machinery) does not appear to be involved in petite mutation induction.

Oestrogens have not conclusively been shown to interact with DNA and very little, if any, mutagenic (nuclear) response has been demonstrated with DES in the yeast $S$. cerevisiae in the absence of oxidizing agents (Mehta and von Borstel, 1982). Therefore, a direct influence even on mitochondrial DNA appears unlikely as a mechanism of petite mutation induction.

On the other hand, membrane structures seem important in petite mutation induction since addition of the membrane-active detergent SDS enabled DESmediated petite mutation induction and increased the effect of Z,Z-DIES. High concentrations of DES have been shown to disturb membranes in liposomes (Weissmann et al., 1976). If mitochondrial membranes are also a target of stilbene oestrogens, petite mutation induction might be due to mitochondrial membrane disturbance, which has been associated with an increase in petite mutants (Jimenez et al., 1988), although the mechanism is not known.

To investigate whether spontaneous petite mutation clones are inherently different to grande clones in sensitivity to the cytostatic and cytotoxic effects of the stilbene oestrogens, four spontaneous petite mutation clones were isolated and tested with $59 \mu \mathrm{g} / \mathrm{ml} \mathrm{3,3^{ \prime } - D E S}$ in parallel with four grande clones of strain D61.M (data not shown). Growth was reduced to $1.2 \%$ (petite mutation clones) and $1.3 \%$ (grande clones) of the control levels and relative viability was reduced to $10 \%$ (petite mutation clones) and $9.2 \%$ (grande clones) of the control levels. The results indicate that petite mutants were induced rather than selected, as cytostatic as well as cytotoxic effects were in the same range for both petite mutation and grande clones. However, since only a small number of colonies can be tested in this way, the

Table 2. Induction of petite mutation colonies and reduction of relative viability by oestrogens under growth conditions with addition of $40 \mu \mathrm{g} / \mathrm{ml}$ sodium dodecylsulphate (SDS). Controls gave $0.22 \%$ petite mutation colonies as an average of five experiments with solvent control and SDS addition and no significant reduction of viability. For other explanations, see legend to Table 1 .

\begin{tabular}{|c|c|c|c|c|c|c|c|c|}
\hline \multirow{2}{*}{$\begin{array}{l}\text { Substance } \\
\text { (in the presence } \\
\text { of SDS) }\end{array}$} & \multicolumn{2}{|c|}{$\begin{array}{l}\text { Concn }(\mu \mathrm{g} / \mathrm{ml}) \text { at } \\
\text { relative viability }\end{array}$} & \multirow{2}{*}{\multicolumn{2}{|c|}{$\begin{array}{l}\text { Relative viability } \\
\text { at concn }(\mu \mathrm{g} / \mathrm{ml}) \\
100 \text { saturated solution }\end{array}$}} & \multicolumn{2}{|c|}{$\begin{array}{l}\text { Petites }(\%) \text { at } \\
\text { relative viability }\end{array}$} & \multirow{2}{*}{\multicolumn{2}{|c|}{$\begin{array}{c}\text { Petites }(\%) \text { at } \\
\text { concn }(\mu \mathrm{g} / \mathrm{ml}) \\
100 \text { saturated solution }\end{array}$}} \\
\hline & \multirow{2}{*}{$\frac{20}{62}$} & \multirow{2}{*}{$\frac{50}{41}$} & & & \multirow{2}{*}{$\frac{20}{9.4}$} & \multirow{2}{*}{$\frac{50}{8.2}$} & & \\
\hline Z,Z-DIES & & & 0.7 & nd & & & 0 & nd \\
\hline DES & 31 & 17 & 2.8 & nd & 9.0 & 4.5 & 25 & nd \\
\hline Stilbene & - & - & 117 & 114 & - & - & 0.3 & 0.2 \\
\hline $17 \beta$-Oestradiol & - & - & 110 & 118 & - & - & 1.2 & 4.8 \\
\hline Oestrone & - & - & 114 & 109 & - & - & 0.1 & 0.2 \\
\hline
\end{tabular}


result can only serve as good evidence and cannot completely rule out the selection of petite mutants.

Stilbene-oestrogens are known to inhibit ATPases (Eilam et al., 1984; McEnery et al., 1989; Pedersen and Carafoli, 1987; Strid et al., 1988). Phenolic hydroxyl groups seem to be essential for interaction with rat liver mitochondria ATPase (McEnery et al., 1989), as was the case in our experiments. To investigate a possible connection between ATPase inhibition and petite mutation induction, several nonhormonal ATPase inhibitors were tested (overnight incubation in growth medium). The bioflavonoid quercetin, an inhibitor of plasma membrane ATPase and mitochondrial ATPase of Neurospora crassa (Bowman et al., 1978), the cardiac glycoside ouabain, an inhibitor of $\mathrm{Na}^{+} / \mathrm{K}^{+}$-ATPases (Pedersen and Carafoli, 1987), N-ethylmaleimid, a covalent SHreactive agent, commonly used as an inhibitor of the V-type ATPases (Pedersen and Carafoli, 1987) and oligomycin, an inhibitor of F-type ATPases (e.g. mitochondrial membrane ATPases; Pedersen and Carafoli, 1987), were used. They were added in various concentrations until cell viability was reduced or saturated solutions were obtained. Up to $40 \mathrm{mg} / \mathrm{ml}$ ouabain was added without achieving either effect. Viability was only decreased by $N$-ethylmaleimid and the frequency of petite mutants was not increased in any case. Involvement of ATPase binding in petite mutation induction seems unlikely in our case. Moreover, a mechanism involving mitochondrial ATPase activation has been suggested for ethidium bromideinduced petite mutation (Mahler and Bastos, 1974).

Another commonly discussed mechanism for petite mutation induction, especially under conditions not permitting growth, is fragmentation of mitochondrial DNA by endonuclease activation. Overnight incubation under conditions not permitting growth (in phosphate buffered saline (PBS) with the petite mutation-inducing oestrogens listed in Table 1) did not result in a significant number of petite mutants (data not shown). Cells were washed and incubated in complete growth medium for $2.5 \mathrm{hr}$ prior to plating. Toxicity was very high under these conditions and might have concealed petite mutation induction. In this experiment berenil produced $100 \%$ petite mutants at a concentration of $1 \mu \mathrm{g} / \mathrm{ml}$ and higher. Shorter incubation with 3,3'-DES in PBS (3.5 hr) and overnight incubation in PBS without additional incubation in growth medium before plating also failed to produce petite mutation. Again, cell toxicity was very high. In general, degradation of DNA should be an energy-consuming process, not being favoured under ATPase inhibitory conditions.

The almost complete inhibition of cell growth was not unexpected because DES is known to induce mitotic arrest in eukaryotes (Hartley-Asp et al., 1985). However, this does not explain the toxicity and petite mutation induction, because mitotic arrest has been attributed to hormone-spindle interaction (Hartley-Asp et al., 1985).

Recent results by Tas et al. (1991) indicate that stilbene oestrogens alter calcium levels in rat glioma cells. Calcium level changes will affect a variety of processes in the cell, possibly including enzyme activity changes, and could indirectly influence mitochondrial DNA.
In conclusion, there is no indication that any of the commonly discussed mechanisms for petite mutation induction, namely direct mitochondrial DNA damage, inhibition of mitochondrial replication or transcription and fragmentation of mitochondrial DNA, apply to stilbene oestrogen-induced respiration deficiency mutagenesis. It is more likely that membrane disturbances and calcium level changes may be involved, but the mechanism is still not clear. Strid et al. (1988) suggested that DES binds in an unspecific manner to hydrophobic entities such as membranes, membrane-bound enzymes and hydrophobic domains of soluble proteins. This could provide a variety of possibilities for harmful cellular interactions.

Stilbene oestrogens are capable of inducing genetic damage at the mitochondrial level in yeast and interacting with mammalian mitochondria. Damage induced in mammalian cells might be more subtle than the drastic changes seen in the yeast system and might be of importance in tumorigenesis, which could either be facilitated by disturbance of mitochondrial function (as recently suggested by Corral et al., 1990; Wunderlich, 1990) or caused by the same mechanisms underlying petite mutagenesis. Further studies concerning the effects of stilbene oestrogens on mitochondria are important for understanding the carcinogenic potential of these hormones.

Acknowledgements-We thank Professor Dr F. K. Zimmermann for providing Saccharomyces cerevisiae D61.M and Mrs E. Stein for expert technical assistance. This study was supported by the Deutsche Forschungsgesellschaft (Sonderforschungsbereich 172).

\section{REFERENCES}

Bowman B. J., Mainzer S. E., Allen K. and Slayman C. W. (1978) Effects of inhibitors on the plasma membrane and mitochondrial adenosine triphosphatases of Neurospora crassa. Biochimica et Biophysica Acta 512, 13-28.

Corral M., Kruh J., Guillonzo C. and Defer N. (1990) Alterations in mitochondrial DNA structure and expression during chemically induced hepatocarcinogenesis. Journal of Cancer Research and Clinical Oncology (Suppl. 116), 1206.

Eilam Y., Lavi H. and Grossowicz N. (1984) Effects of inhibitors of plasma-membrane ATPases on potassium and calcium fluxes, membrane potential and proton motive force in the yeast Saccharomyces cerevisiae. Microbios 41 (165-166), 177-189.

Epe B., Harttig U., Stopper H. and Metzler M. (1990) Covalent binding of reactive estrogen metabolites to microtubular protein as a possible mechanism of aneuploidy induction and neoplastic cell transformation. Environmental Health Perspectives 88, 123-127.

Hartley-Asp B., Deinum J. and Wallin M. (1985) Diethylstilbestrol induces metaphase arrest and inhibits microtubule assembly. Mutation Research 143, 231-234.

IARC (1979) Monographs on the Evaluation of the Carcinogenic Risk of Chemicals to Humans. Vol. 21. Sex Hormones (II). International Agency for Research on Cancer, Lyon.

Jimenez J., Longo E. and Benito T. (1988) Induction of petite yeast mutants by membrane active agents. Applied and Environmental Microbiology 54 (12), 3126-3132.

McEnery M. W., Hullihen J. and Pedersen P. L. (1989) Fo "Proton channel" of rat liver mitochondria. Journal of Biological Chemistry 264 (20), 12029-12036. 
McLachlan J. A., Wong A., Degen G. H. and Barrett J. C. (1982) Morphological and neoplastic transformation of Syrian hamster embryo fibroblasts by diethylstilbestrol and analogs. Cancer Research 42, 3040-3045.

Mahler H. R. and Bastos R. N. (1974) Coupling between mitochondrial mutation and energy transduction. Proceedings of the National Academy of Sciences of the U.S.A. 71, 2241-2245.

Mahler H. R. and Perlman P. S. (1973) Induction of respiratory deficient mutants in Saccharomyces cerevisiae by berenil. Molecular and General Genetics 121, 285-294.

Mehta R. D. and von Borstel R. C. (1982) Genetic activity of diethylstilbestrol in Saccharomyces cerevisiae: enhancement of mutagenicity by oxidizing agents. Mutation Research 92, 49-61.

Metzler M. (1984) Metabolism of stilbene estrogens and steroidal estrogens in relation to carcinogenicity. Archives of Toxicology 55, 104-109.

Pedersen P. L. and Carafoli E. (1987) Ion motive ATPases: ubiquity, properties, and significance to cell function. Trends in Biochemical Science 12, 146-150.

Strid A., Nyren P. and Baltscheffsky M. (1988) Diethylstilbestrol, interactions with membrane and proteins and the different effect upon $\mathrm{Ca}_{2}{ }^{+}$and $\mathrm{Mg}_{2}{ }^{+}$dependent activities of the F1-ATPase from Rhodospirillum rubrum. European Journal of Biochemistry 176, 281-285.

Tas P., Stopper H., Koschel K. and Schiffmann D. (1991) Influence of the carcinogenic oestrogen diethylstilboestrol on the intracellular calcium level in C6 rat glioma cells. Toxicology in Vitro 5, 463-465.

Tsutsui T., Suzuki N., Fukuda S., Sado M., Maizumi H., McLachlan J. A. and Barrett J. C. (1987) 17ß-Estradiol induced cell transformation and aneuploidy of Syrian hamster embryo cells in culture. Carcinogenesis 8, 1715-1719.

Weissmann G., Collins T., Evers A. and Dunham B. (1976) Membrane perturbation: studies employing a calciumsensitive dye, arsenazo III, in liposomes. Proceedings of the National Academy of Sciences of the U.S.A. 73, $510-514$.

Wunderlich V. (1990) Mitochondrial DNA in human disease: a summary of recent developments. Journal of Cancer Research and Clinical Oncology (Suppl. 116), 1206.

Zimmermann F. K. and Scheel I. (1984) Genetic effects of 5-azacytidine in Saccharomyces cerevisiae. Mutation Research 139, 21-24. 